\title{
Collisional Properties of a Polarized Fermi Gas with Resonant Interactions
}

\author{
G. M. Bruun, ${ }^{1,2}$ A. Recati, ${ }^{1}$ C. J. Pethick,,${ }^{2,3}$ H. Smith,${ }^{2}$ and S. Stringari ${ }^{1}$ \\ ${ }^{1}$ Dipartimento di Fisica, Università di Trento and CNR-INFM BEC Center, I-38050 Povo, Trento, Italy \\ ${ }^{2}$ Niels Bohr Institute, University of Copenhagen, DK-2100 Copenhagen Ø, Denmark \\ ${ }^{3}$ NORDITA, Roslagstullsbacken 21, 10691 Stockholm, Sweden
}

\begin{abstract}
Highly polarized mixtures of atomic Fermi gases constitute a novel Fermi liquid. We demonstrate how information on thermodynamic properties may be used to calculate quasiparticle scattering amplitudes even when the interaction is resonant and apply the results to evaluate the damping of the spin dipole mode. We estimate that under current experimental conditions, the mode would be intermediate between the hydrodynamic and collisionless limits.
\end{abstract}

Recent experiments with polarized atomic Fermi gases have made possible the realization of novel quantum systems [1]. The case we shall focus on here is that of a highly polarized mixture of two fermion species. Because the system remains normal at the lowest temperatures attained, it is a completely new normal Fermi liquid. Since the interaction may be tuned by exploiting Feshbach resonances, it is possible to investigate the effects of strong correlations in a previously inaccessible regime [2]. The system is particularly rich, because of the ability to vary the relative numbers of the two sorts of atom and the ratio of the atomic masses, in addition to the strength of the interaction and the temperature. In this Letter we calculate how the resonant interaction affects the frequency and damping of dipole modes in which the two components move relative to each other. A key element in the calculation is the use of thermodynamic arguments to deduce quasiparticle scattering amplitudes when the gas is strongly interacting. Dipole modes have previously been studied for two different spin states of ${ }^{40} \mathrm{~K}$ at higher temperatures in a regime in which the gas is sufficiently dilute that the scattering amplitude is simply related to the scattering length [3]. Related issues have been investigated in the context of spin-drag phenomena in low-dimensional Fermi systems [4, 5].

We consider a homogeneous gas of two species of fermion, which may be either two different hyperfine states of the same atom or two different atoms, e.g., ${ }^{6} \mathrm{Li}$ and ${ }^{40} \mathrm{~K}$. We denote the species by the label $\sigma=\uparrow, \downarrow$, the numbers of atoms by $N_{\uparrow}$ and $N_{\downarrow}$, and their masses by $m_{\uparrow}$ and $m_{\downarrow}$. The interaction between an up-atom and a down-atom is characterized by the $s$-wave scattering length $a$. Interactions between like atoms may be neglected because the $s$-wave component vanishes due to the Pauli principle. In the case of large polarization or population imbalance, $N_{\uparrow} \gg N_{\downarrow}$, the majority (up) component is essentially an ideal Fermi gas with an effective mass $m_{\uparrow}^{*}$ equal to the bare mass $m_{\uparrow}$ even in the unitarity limit where $|a| \rightarrow \infty$. By contrast, the minority (down) component is strongly affected by the interaction with the up-atoms. The ground state energy of a single down-atom in a sea of up-atoms can be written as

$$
\mu_{\downarrow}=-\alpha \epsilon_{\mathrm{F} \uparrow},
$$

$\epsilon_{\mathrm{F} \uparrow}=\left(6 \pi^{2} n_{\uparrow}\right)^{2 / 3} / 2 m_{\uparrow}$ being the Fermi energy of the spin-up component and $n_{\sigma}$ the density of the $\sigma$ atoms (we use $\hbar=1)$. The parameter $\alpha$ depends on the mass ratio $m_{\downarrow} / m_{\uparrow}$ and on the variable $k_{\mathrm{F} \uparrow} a$, where $k_{\mathrm{F} \uparrow}$ is the Fermi momentum of the up-atoms. For equal masses, Monte Carlo calculations in the unitarity limit give $\alpha \approx 0.6$ [6, 7, 8], and for other mass ratios $\alpha$ has been evaluated in the ladder approximation [9] which, for equal masses, gives good agreement with the Monte Carlo results. It is found that $\alpha$ is an increasing function of $m_{\downarrow} / m_{\uparrow}$. The effective mass $m_{\downarrow}^{*}$ of a down-atom is different from the bare mass and, for $m_{\downarrow}=m_{\uparrow}$, Monte Carlo calculations in the unitarity limit give $m_{\downarrow}^{*} \approx m_{\downarrow}[6]$. Furthermore, the ladder-approximation calculations show that for large $|a|$ the single-particle propagator of the minority component has a large quasiparticle peak [10].

The damping of counterflow is determined by the rate at which momentum is transferred between the two components. Consider a situation in which the two components are spatially uniform. We use concepts of Fermi liquid theory to describe the effects of the interactions. The system is considered as an ideal gas of majority (up) atoms mixed with a gas of minority (down) atoms whose elementary excitations are quasiparticles with effective mass $m_{\downarrow}^{*}$. We take the minority component to have a mean velocity $\mathbf{v}$ with respect to the majority component giving a total momentum per unit volume $\mathbf{P}_{\downarrow}=n_{\downarrow} m_{\downarrow}^{*} \mathbf{v}$.

We define a momentum relaxation time $\tau_{P}$ by the relation

$$
\frac{d \mathbf{P}_{\downarrow}}{d t}=-\frac{\mathbf{P}_{\downarrow}}{\tau_{P}},
$$

and we shall calculate $\tau_{P}$ by assuming that both components are in thermal equilibrium described by the distribution functions $n_{\mathbf{p}^{\prime} \uparrow}=f\left[\beta\left(\epsilon_{\mathbf{p}^{\prime} \uparrow}-\mu_{\uparrow}\right)\right]$ and $n_{\mathbf{p} \downarrow}=$ $f\left[\beta\left(\epsilon_{\mathbf{p} \downarrow}-\mathbf{p} \cdot \mathbf{v}-\mu_{\downarrow}\right)\right]$ with $\beta=1 / k T$ and $f(x)=1 /\left(\mathrm{e}^{x}+1\right)$. The single particle energies are $\epsilon_{\mathbf{p}^{\prime} \uparrow}=p^{\prime 2} / 2 m_{\uparrow}$ and $\epsilon_{\mathbf{p} \downarrow}=p^{2} / 2 m_{\downarrow}^{*}$. The term $\mathbf{p} \cdot \mathbf{v}$ boosts the down-atom distribution function by a velocity $\mathbf{v}$. The rate of change of the momentum of the down-atoms due to their colli- 
sions with up-atoms may then be written as

$$
\begin{aligned}
\frac{d \mathbf{P}_{\downarrow}}{d t}=-2 \pi & \frac{|U|^{2}}{V^{3}} \sum_{\mathbf{p}, \mathbf{p}^{\prime}, \mathbf{q}} \mathbf{p}\left[n_{\mathbf{p} \downarrow} n_{\mathbf{p}^{\prime} \uparrow}\left(1-n_{\mathbf{p}-\mathbf{q} \downarrow}\right)\left(1-n_{\mathbf{p}^{\prime}+\mathbf{q} \uparrow}\right)\right. \\
& \left.-n_{\mathbf{p}-\mathbf{q} \downarrow} n_{\mathbf{p}^{\prime}+\mathbf{q} \uparrow}\left(1-n_{\mathbf{p} \downarrow}\right)\left(1-n_{\mathbf{p}^{\prime} \uparrow}\right)\right] \\
& \times \delta\left(\epsilon_{\mathbf{p} \downarrow}+\epsilon_{\mathbf{p}^{\prime} \uparrow}-\epsilon_{\mathbf{p}-\mathbf{q} \downarrow}-\epsilon_{\mathbf{p}^{\prime}+\mathbf{q} \uparrow}\right),
\end{aligned}
$$

where $V$ is the volume of the system. The two terms in (3) correspond to a pair of quasiparticles with momenta $\mathbf{p}$ and $\mathbf{p}^{\prime}$ scattering to a pair with momenta $\mathbf{p}-\mathbf{q}$ and $\mathbf{p}^{\prime}+\mathbf{q}$ and the inverse process.

The effective interaction $U$ may be estimated from thermodynamic arguments. The Landau quasiparticle interaction averaged over the angle betwen the momenta of the two quasiparticles may be determined from the energy as a function of the densities of the two components, $f_{\uparrow \downarrow}^{0}=\partial^{2} E / \partial n_{\uparrow} \partial n_{\downarrow}=\partial \mu_{\downarrow} / \partial n_{\uparrow}$, where $E$ is the energy density of the system. Since the momenta of the down-atoms are assumed to be much less than the Fermi momentum of the up-atoms, the quasiparticle interaction may be taken to be independent of the angle between the quasiparticle momenta. To estimate scattering amplitudes in terms of Landau parameters it is generally necessary to allow for additional processes due to screening by particle-hole pairs [11]. However, since we assume that $n_{\downarrow} \ll n_{\uparrow}$, these processes may be neglected, and we take the scattering amplitude to be independent of the direction of the momenta of the quasiparticles and equal to

$$
U=\frac{\partial \mu_{\downarrow}}{\partial n_{\uparrow}}=\frac{2 \pi^{2}}{m_{\uparrow} k_{\mathrm{F} \uparrow}} \gamma,
$$

where, from Eq. (1), $\gamma=-\alpha\left[1+(3 / 2) \partial \ln \alpha / \partial \ln n_{\uparrow}\right]$ and $k_{\mathrm{F} \sigma}=\left(6 \pi^{2} n_{\sigma}\right)^{1 / 3}$. For the case of a resonant interaction, $\gamma=-\alpha$ and $U=-(2 \alpha / 3) \epsilon_{\mathrm{F} \uparrow} / n_{\uparrow} \propto 1 / k_{\mathrm{F} \uparrow}$. This is very different from the effective interaction at low densities, which is proportional to $a$.

It is convenient to rewrite the expression (3) in terms of response functions. On introducing the quantity $\omega_{\mathbf{q}}=\mathbf{q} \cdot \mathbf{v}$, using the relation $n_{\mathbf{p}}\left(1-n_{\mathbf{p}-\mathbf{q}}\right)=\left(n_{\mathbf{p}}-\right.$ $\left.n_{\mathbf{p}-\mathbf{q}}\right) /\left\{1-\exp \left[\beta\left(\epsilon_{\mathbf{p}}-\epsilon_{\mathbf{p}-\mathbf{q}}\right)\right]\right\}$, and taking the continuum limit we obtain

$$
\begin{gathered}
\frac{d \mathbf{P}_{\downarrow}}{d t}=-2 \pi|U|^{2} \int \frac{d^{3} q}{(2 \pi)^{3}} \mathbf{q} \\
\times \int_{-\infty}^{\infty} d \omega \frac{\operatorname{Im} \chi_{\downarrow}\left(q, \omega_{\mathbf{q}}-\omega\right) \operatorname{Im} \chi_{\uparrow}(q, \omega)}{\left(1-\mathrm{e}^{\beta\left(\omega-\omega_{\mathbf{q}}\right)}\right)\left(1-\mathrm{e}^{-\beta \omega}\right)}
\end{gathered}
$$

where

$$
\operatorname{Im} \chi_{\sigma}(q, \omega)=\int \frac{d^{3} p}{(2 \pi)^{3}}\left(n_{\mathbf{p} \sigma}-n_{\mathbf{p}+\mathbf{q} \sigma}\right) \delta\left(\omega+\epsilon_{\mathbf{p} \sigma}-\epsilon_{\mathbf{p}+\mathbf{q} \sigma}\right)
$$

is, apart from a factor of $\pi$, the imaginary part of the Lindhard function, and the distribution functions are

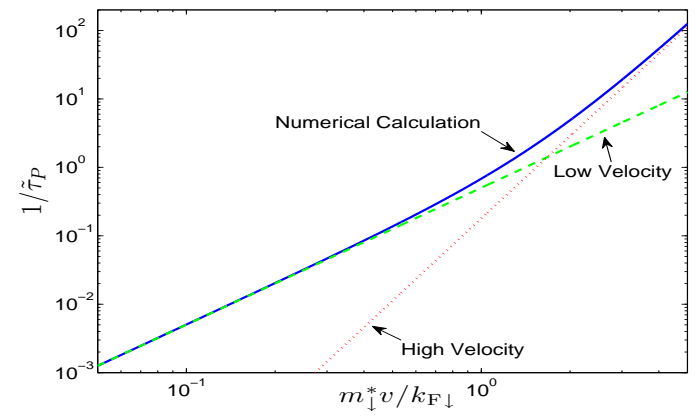

FIG. 1: The scaled momentum relaxation rate $1 / \tilde{\tau}_{P}$ at $T=0$ versus relative velocity $v$ in units of $k_{\mathrm{F} \downarrow} / m_{\downarrow}^{*}$. The full line is the result of numerical integration of Eq. (5), the dashed line is low-velocity result (7) and the dotted line is the highvelocity result (8).

now global equilibrium ones without the boost for the down-atoms.

Let us consider first the momentum relaxation rate for $T=0$. In this case, the Bose factors in (5) result in the condition $0 \leq \omega \leq \omega_{\mathbf{q}}$. In the following we discuss two important limiting regimes where simple expressions for $\tau_{P}$ can be obtained.

(i) The low velocity regime, $m_{\downarrow}^{*} v \ll k_{\mathrm{F} \downarrow}$. In this case the significant contribution to (5) comes from $q \leq 2 k_{\mathrm{F} \downarrow}$ with a small energy transfer $\omega_{\mathbf{q}} \ll k_{\mathrm{F} \downarrow}^{2} / 2 m_{\downarrow}^{*}$. We can then use $\operatorname{Im} \chi_{\sigma}(q, \omega)=m_{\sigma}^{* 2} \omega /\left(4 \pi^{2} q\right)$ and the resulting integrals in (5) yield

$$
\frac{1}{\tau_{P}}=\frac{4 \pi}{25}|\gamma|^{2}\left(\frac{k_{\mathrm{F} \downarrow}}{k_{\mathrm{F} \uparrow}}\right)^{2} m_{\downarrow}^{*} v^{2}=\frac{4 \pi}{25} \frac{1}{\tau_{0}}\left(\frac{m_{\downarrow}^{*} v}{k_{\mathrm{F} \downarrow}}\right)^{2},
$$

where $1 / \tau_{0}=|\gamma|^{2} k_{\mathrm{F} \downarrow}^{4} / m_{\downarrow}^{*} k_{\mathrm{F} \uparrow}^{2}$.

(ii) The high velocity regime, $k_{\mathrm{F} \downarrow} \ll m_{\downarrow}^{*} v \ll k_{\mathrm{F} \uparrow}$. In this case we can again carry out the integrations in (5) and obtain

$$
\frac{1}{\tau_{P}}=\frac{2 \pi}{35}|\gamma|^{2} \frac{m_{\downarrow}^{* 3} v^{4}}{k_{\mathrm{F} \uparrow}^{2}}=\frac{2 \pi}{35} \frac{1}{\tau_{0}}\left(\frac{m_{\downarrow}^{*} v}{k_{\mathrm{F} \downarrow}}\right)^{4} .
$$

More generally, the scaled relaxation time $\tilde{\tau}_{P} \equiv \tau_{P} / \tau_{0}$ depends only on the variable $\tilde{v}=m_{\downarrow}^{*} v / k_{\mathrm{F} \downarrow}$ provided $m_{\downarrow}^{*} v \ll k_{\mathrm{F} \uparrow}$.

In Fig. 1, we plot the $T=0$ momentum relaxation rate $1 / \tilde{\tau}_{P}$ calculated numerically from (5) as a function of velocity. For the numerical calculations we took $m_{\downarrow}^{*} / m_{\uparrow}=1$ and $k_{\mathrm{F} \downarrow} / k_{\mathrm{F} \uparrow}=0.1$. The relaxation rate increases with increasing $v$ because the available phase space for scattering grows.

We now turn to non-zero temperature. Current experiments on highly polarized gases achieve very low temperatures and we therefore first analyze the regime $T \ll T_{\mathrm{F} \downarrow} \ll T_{\mathrm{F} \uparrow}$, in which both components are degenerate. Here $k T_{\mathrm{F} \downarrow}=k_{\mathrm{F} \downarrow}^{2} / 2 m_{\downarrow}^{*}$ and $k T_{\mathrm{F} \uparrow}=k_{\mathrm{F} \uparrow}^{2} / 2 m_{\uparrow}$. Furthermore, for small relative velocities, $v k_{\mathrm{F} \downarrow} \ll k T$, it 
is sufficient to expand the integrand in (5) to first order in $\beta \omega_{\mathbf{q}}$. Using the symmetry property $\operatorname{Im} \chi_{\sigma}(q, \omega)=$ $-\operatorname{Im} \chi_{\sigma}(q,-\omega)$ we obtain

$$
\begin{array}{r}
\frac{d \mathbf{P}_{\downarrow}}{d t}=-\mathbf{v} \frac{\pi|U|^{2}}{3 k T} \int \frac{d^{3} q}{(2 \pi)^{3}} q^{2} \\
\times \int_{-\infty}^{\infty} d \omega \frac{\operatorname{Im} \chi_{\downarrow}(q,-\omega) \operatorname{Im} \chi_{\uparrow}(q, \omega)}{\left(1-\mathrm{e}^{\beta \omega}\right)\left(1-\mathrm{e}^{-\beta \omega}\right)} .
\end{array}
$$

For $T \ll T_{\mathrm{F} \downarrow}$, we can again use the result $\operatorname{Im} \chi_{\sigma}(q, \omega)=$ $m_{\sigma}^{* 2} \omega /\left(4 \pi^{2} q\right)$ which yields for the relaxation rate in the limit of low velocities the expression

$$
\frac{1}{\tau_{P}}=\frac{4 \pi^{3}}{9}|\gamma|^{2} \frac{m_{\downarrow}^{*}}{k_{\mathrm{F} \uparrow}^{2}}(k T)^{2}=\frac{\pi^{3}}{9} \frac{1}{\tau_{0}}\left(\frac{T}{T_{\mathrm{F} \downarrow}}\right)^{2} .
$$

The $T^{2}$-dependence is due to the fact that the phase space for scattering increases with temperature. Equation (10) shows that for equal masses of the two components and at unitarity $1 / \tau_{P} \sim k T^{2} / T_{F \uparrow}$, as one would expect on dimensional grounds because the effective interaction measured in terms of the density of states of the up-atoms is of order unity.

Next we discuss the behavior at temperatures comparable with or higher than $T_{\mathrm{F} \downarrow}$. When the masses of the two components are very different it becomes relevant to consider also temperature scales characterized by $T_{0} \equiv\left(m_{\uparrow} / m_{\downarrow}\right) T_{\mathrm{F} \uparrow}$. As an example let us consider the case when $T_{\mathrm{F} \downarrow} \ll T_{0} \ll T_{\mathrm{F} \uparrow}$. In the classical regime for the minority population $\left(T \gg T_{\mathrm{F} \downarrow}\right)$ we have

$\frac{\operatorname{Im} \chi_{\downarrow}}{n_{\downarrow}}=\left(\frac{2 \pi m_{\downarrow}^{*}}{k T q^{2}}\right)^{1 / 2} \mathrm{e}^{-\omega^{2} m_{\downarrow}^{*} / 2 q^{2} k T-q^{2} / 8 m_{\downarrow}^{*} k T} \sinh \left(\frac{\omega}{2 k T}\right)$.

We treat two limiting cases: a) $T_{\mathrm{F} \downarrow} \ll T \ll T_{0}$. Here the upper limit on the $q$-integration in (9) may be extended to infinity and the result of carrying out the integrations yields again a $T^{2}$-dependence for $1 / \tau_{P}$, which differs from (10) only by the replacement of $\pi^{3} / 9 \approx 3.45$ by 2.98 . This suggests that the low-temperature result (10) is accurate over a much wider temperature range. b) $T_{\mathrm{F} \downarrow} \ll T_{0} \ll$ $T \ll T_{\mathrm{F} \downarrow}$. Here the upper limit on the $q$-integration is $2 k_{\mathrm{F} \uparrow}$. Using the fact that $\omega / \sinh (\omega / 2 k T)$ may be approximated by $2 k T$ and $\exp \left(-q^{2} / 8 m_{\downarrow}^{*} k T\right)$ by 1 we obtain, by integrating first over $\omega$ and subsequently over $q$, the expression $m_{\downarrow}^{*} / \tau_{P}=|U|^{2} m_{\uparrow}^{2} k_{\mathrm{F} \uparrow}^{4} / 6 \pi^{3}$. For a dilute system with $m_{\downarrow}^{*} \gg m_{\uparrow}$ the effective interaction is $U=2 \pi a / m_{\uparrow}$ and this result becomes $m_{\downarrow}^{*} / \tau_{P}=k_{\mathrm{F} \uparrow} n_{\uparrow} \sigma$, in agreement with the known result for the high-temperature mobility of a heavy particle in a degenerate quantum gas, the cross section being $\sigma=4 \pi a^{2}$.

Experimental considerations We now relate our results for the homogeneous case to experimentally observable features in the presence of a trapping potential $V_{\sigma}$, which will in general be different for the two species. The momentum relaxation rate is most directly probed by exciting the spin dipole mode of a Fermi gas above the critical polarization where the system is normal at all temperatures [1, 6]. Let us assume that the cloud of down-atoms is displaced by a distance $\delta X$ from the equilibrium position in the harmonic trap. Depending on the amplitude of the displacement (and consequently on the velocity acquired by the minority component due to the external force) as well as on the value of temperature, the cloud either oscillates with weak damping around $\delta X=0$ (collisionless regime) or it relaxes towards equilibrium without any oscillations (hydrodynamic regime).

In the collisionless limit the frequency of the oscillation is readily obtained in the case of large imbalance where it is sufficient to consider the single quasiparticle Hamiltonian to describe the motion of the minority component [12]. The interaction energy of a down-atom is $-\alpha \epsilon_{\mathrm{F} \uparrow}$. In the Thomas-Fermi approximation, $\epsilon_{\mathrm{F} \uparrow}+V_{\uparrow}$ is a constant. Thus, the total potential felt by a down-atom is $V_{\downarrow}+\alpha V_{\uparrow}$. The Hamiltonian for a single down-atom then has the form $H_{\mathrm{sp}}=p^{2} / 2 m_{\downarrow}^{*}+V_{\downarrow}+\alpha V_{\uparrow}$. The interaction with the majority component is taken into account through the effective mass $m_{\downarrow}^{*}$ and the change in the potential caused by the interaction with the up-atoms. For simplicity we restrict ourselves to a resonant interaction, in which case $\alpha$ is independent of density and from this Hamiltonian, the frequency $\omega_{\mathrm{D}}$ of the spin dipole mode for a harmonic trap is easily calculated to be $\underline{6}, 12$ ]

$$
\omega_{\mathrm{D}}=\omega_{\downarrow} \sqrt{\frac{m_{\downarrow}}{m_{\downarrow}^{*}}\left(1+\frac{m_{\uparrow} \omega_{\uparrow}^{2}}{m_{\downarrow} \omega_{\downarrow}^{2}} \alpha\right)},
$$

where $\omega_{\sigma}$ is the oscillation frequency in the trap for species $\sigma$. Measurements of the spin dipole frequency thus provide a unique opportunity to test directly the effects of interactions which, according to the theoretical estimates of $\alpha$ and $m_{\downarrow}^{*}$, should increase the value of the frequency by a factor 1.23 when the trapping potential is harmonic and the same for the two species. The spin dipole mode, however, is well defined only in the collisionless limit $\omega_{\mathrm{D}} \tau_{P} \gg 1$. It becomes overdamped in the hydrodynamic regime $\omega_{\mathrm{D}} \tau_{P} \ll 1$ since the spin current is not conserved by collisions [13].

In order to estimate whether under current experimental conditions the spin dipole mode will be in the hydrodynamic or in the collisionless regime, we calculate $\omega_{\mathrm{D}} \tau_{P}$. It is convenient to express results in terms of the amplitude of the displacement of the down-atom cloud $\delta X$, which is controllable experimentally. We shall assume that the displacement of the down-atom cloud is sufficiently small ( $\delta X \ll R_{\uparrow}$ where $R_{\uparrow}$ is the radius of the majority cloud) that the density of up-atoms may be regarded as uniform when estimating the relaxation rate. The relative velocity of the two components is given by $v=\omega_{\mathrm{D}} \delta X$. We shall adopt the values $N_{\uparrow}=10^{7}$, and $N_{\downarrow} / N_{\uparrow}=0.026\left(T_{\mathrm{F} \downarrow} / T_{\mathrm{F} \uparrow}=0.3\right)$ corresponding to conditions achieved in the MIT experiment [1] for a mixture 


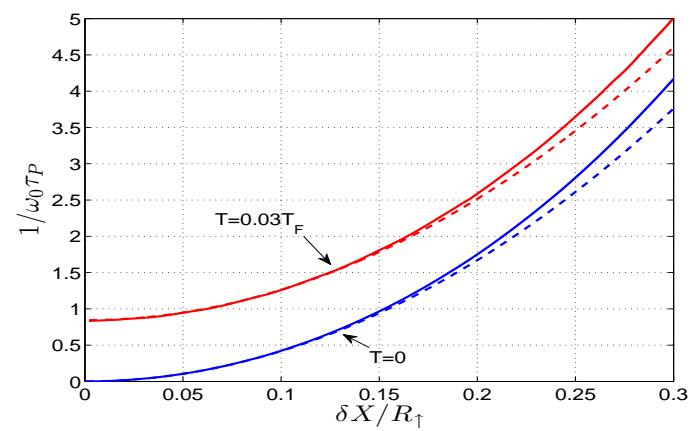

FIG. 2: The quantity $1 / \omega_{0} \tau_{P}$ determining the damping of the dipole mode as a function of the amplitude of the oscillation for $T=0$ and $T=0.03 T_{\mathrm{F} \uparrow}$. (For details see text.)

of ${ }^{6} \mathrm{Li}$-atoms in two different hyperfine states, together with the values $\alpha=0.6$ and $m_{\downarrow}^{*} / m_{\downarrow} \approx 1$ obtained theoretically [6, 9]. We approximate $\omega_{\mathrm{D}}$ by the trap frequency $\omega_{0}$, which we take to be the same for both species. The lower full line in Fig. 2 shows $1 / \omega_{0} \tau_{P}$ as a function of $\delta X / R_{\uparrow}$ obtained from Eq. (5) by numerical integration for $T=0$, while the lower dashed line is the expression (77). The upper lines are for a temperature $T=0.03 T_{\mathrm{F} \uparrow}$, the full one being the result of a numerical calculation and the dashed line is the sum of the results (7) and (10) which, expressed in terms of the number of up-atoms, are

$$
\frac{1}{\omega_{0} \tau_{P}}=\frac{8 \pi}{25}\left(6 N_{\uparrow}\right)^{1 / 3} \alpha^{2} \frac{m_{\downarrow}^{*}}{m_{\uparrow}}\left(\frac{T_{\mathrm{F} \downarrow}}{T_{\mathrm{F} \uparrow}}\right)^{2}\left(\frac{\delta X}{R_{\uparrow}}\right)^{2}
$$

and

$$
\frac{1}{\omega_{0} \tau_{P}}=\frac{2 \pi^{3}}{9}\left(6 N_{\uparrow}\right)^{1 / 3} \alpha^{2} \frac{m_{\downarrow}^{*}}{m_{\uparrow}}\left(\frac{T}{T_{\mathrm{F} \uparrow}}\right)^{2}
$$

where we have used the result $\gamma=-\alpha$ for a resonant interaction and the fact that $k T_{\mathrm{F} \uparrow}=k_{\mathrm{F} \uparrow}^{2} / 2 m_{\uparrow}=$ $\left(6 N_{\uparrow}\right)^{1 / 3} \omega_{0}$. The plots demonstrate that the analytical results are a good approximation to those obtained by direct numerical integration in the regimes of experimental interest. The sum of the results (7) and (8), which is not shown, is an even better approximation to the numerical results.

The calculated values of $\omega_{0} \tau_{P}$ demonstrate that, for the experimental conditions now attainable at MIT, the polarized normal phase is in a regime intermediate between collisionless and hydrodynamic behavior, implying significant damping of the spin dipole mode. At lower temperature, the gas enters the collisionless regime.

How important collisions are in a given mode is sensitive to the anisotropy of the trap, which we have neglected so far. For instance, for a cigar-shaped trap $\left(\omega_{z}<\omega_{\perp}\right)$ the transverse mode will be more collisionless, the value of $1 / \omega_{\mathrm{D}} \tau_{P}$ being multiplied by a factor $\left(\omega_{z} / \omega_{\perp}\right)^{1 / 3}$, for a fixed value of $\left(\omega_{\perp}^{2} \omega_{z}\right)^{1 / 3}$. When the two species are different elements, the value of $\omega_{0} \tau_{P}$ will be depend on the trapping potentials of the two species, which can be varied independently of each other.

For low velocity, $m_{\downarrow}^{*} v \ll k_{\mathrm{F} \downarrow}$, one sees from (7) and (10) that the momentum relaxation rate scales as $m_{\downarrow}^{*}$. Consequently, since $m_{\downarrow}^{*} \approx m_{\downarrow}$ the spin motion can be made more collisionless by trapping an atom mixture with a lighter minority component. However, calculations indicate that this effect is reduced due to the fact that, at unitarity, the scattering amplitude for the case of extreme imbalance increases with decreasing $m_{\downarrow} / m_{\uparrow}<1[9]$. For $m_{\downarrow} / m_{\uparrow}>1$ the scattering amplitude is predicted to be approximately constant and therefore $1 / \tau_{P} \propto m_{\downarrow}$ in this regime. Thus, the spin motion becomes more hydrodynamic for $m_{\downarrow} / m_{\uparrow}>1$. It would be interesting to test these predictions experimentally.

In conclusion, we have demonstrated that for a strongly polarized atomic gas with resonant interactions, scattering amplitudes exhibit a universal behavior, just as thermodynamic properties do 14]. Predictions for the damping of the spin dipole mode have been presented, and it would be valuable to make measurements of the mode. Our approach may be extended to less highly polarized gases by including the effects of screening by the minority component. We acknowledge fruitful discussions with L. P. Pitaevskii. C. J. P. is grateful to

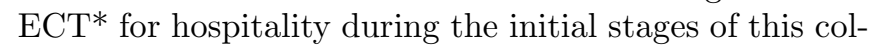
laboration. A. R. and S. S. acknowledge support from the Euroquam FERMIX program.

[1] Y. Shin, M. W. Zwierlein, C. H. Schunck, A. Schirotzek, and W. Ketterle, Phys. Rev. Lett. 97, 030401 (2006); Y. Shin, C. H. Schunck, A. Schirotzek, and W. Ketterle, Nature 451, 689 (2008).

[2] For a review of atomic Fermi gases see, e.g., S. Giorgini, L. P. Pitaevskii, and S. Stringari, arXiv:0706.3360

[3] S. D. Gensemer and D. S. Jin, Phys. Rev. Lett. 87, 173201 (2001); B. DeMarco and D. S. Jin, Phys. Rev. Lett. 88, 040405 (2002).

[4] C. P. Weber et al., Nature 437, 1330 (2005).

[5] See, e.g., M. Polini and G. Vignale, Phys. Rev. Lett. 98, 266403 (2007) and references therein.

[6] C. Lobo, A. Recati, S. Giorgini, and S. Stringari, Phys. Rev. Lett. 97, 200403 (2006).

[7] S. Pilati and S. Giorgini, arXiv:0710.1549.

[8] N. Prokof'ev and B. Svistunov, Phys. Rev. B 77, 020408(R) (2008).

[9] R. Combescot, A. Recati, C. Lobo, and F. Chevy, Phys. Rev. Lett. 98, 180402 (2007).

[10] P. Massignan, G. M. Bruun, and H. T. C. Stoof, Phys. Rev. A 77, 031601(R) (2008).

[11] G. Baym and C. J. Pethick, Landau Fermi-liquid Theory: Concepts and Applications (Wiley, New York, 1991).

[12] A. Recati, C. Lobo, and S. Stringari, arXiv:0803.4419.

[13] L. Vichi and S. Stringari, Phys. Rev. A 60, 4734 (1999).

[14] T. L. Ho, Phys. Rev. Lett. 92, 090402 (2004). 